\title{
The little hierarchy problem for new physics just beyond the LHC
}

\author{
F. Bazzocchi ${ }^{\dagger \ddagger}$ and M. Fabbrichesi ${ }^{\ddagger}$ \\ ${ }^{\ddagger}$ INFN, Sezione di Trieste and \\ ${ }^{\dagger}$ SISSA, via Bonomea 265, 34136 Trieste, Italy
}

(Dated: June 11, 2018)

\begin{abstract}
We discuss two possible extensions to the standard model in which an inert singlet scalar state that only interacts with the Higgs boson is added together with some fermions. In one model the fermions provide for a see-saw mechanism for the neutrino masses, in the other model for grand unification of the gauge couplings. Masses and interaction strengths are fixed by the requirement of controlling the finite one-loop corrections to the Higgs boson mass thus addressing the little hierarchy problem. The inert scalar could provide a viable dark matter candidate. Direct detection of this scalar singlet in nuclear scattering experiments is possible with a cross section within reach of future experiments.
\end{abstract}

PACS numbers: $11.30 . \mathrm{Qc}, 12.60 \mathrm{Fr}, 14.80 . \mathrm{Bn}, 95.35 .+\mathrm{d}$

1. If the absence of new states below the TeV scale 1 will be confirmed as the integrated luminosity of the LHC increases in the next few years, it will become unfortunately necessary to move the scale at which to expect new physics outside the reach of the experiments. Such an higher scale is somewhat in agreement with what already found at LEP where a cutoff scale for higher order operators encoding new physics is found to be larger than $5 \mathrm{TeV}[2$. Recent fits of supersymmetric models [3] also indicate that the masses of the new particles may be just beyond the LHC reach and between 5 and $10 \mathrm{TeV}$.

The presence of new physics above $5 \mathrm{TeV}$ rises the problem of the little hierarchy: For the Higgs boson mass [4] (and the electroweak (EW) vacuum expectation value) to be in the $100 \mathrm{GeV}$ range - that is, roughly between one and two orders of magnitude smaller than the new physics scale - renormalization effects must at least partially cancel out in order to prevent the Higgs boson mass from shifting to the higher energy scale.

One may implement such a cancellation by an appropriated choice of the Higgs boson bare mass but this would imply a fine-tuning of such a counter-term in which low and high-energy degrees of freedom are mixed. A more natural choice requires that the cancellation occurs at the higher scale and either comes from a symmetry or is an accident in which the various terms conspire to cancel against each other. In the latter case, the cancellation is best thought as the effect of a dynamical mechanism, at work at the high energy scale, which arises from new physics that we do not know. The built-in fine-tuning of such a conspiracy (the same we would have at the level of the counter-terms) is of the order of the ratio of the two energy scales, in our case of about $10 \%$.

In what follows, we come back to the little hierarchy problem by following the empirical approach and discuss two possible scenarios for new physics: a representative see-saw model for neutrino masses and a grand unification model. In both of them, the addition of new states would shift the Higgs boson mass to the new scale unless we balance the new contributions to prevent large oneloop renormalizations. The identification of what states (their masses and couplings to the Higgs boson) must be present for such a balancing act to occur provides the heuristic power of the little hierarchy problem.

While many possible new states can be added to prevent large corrections to the Higgs boson mass, the simplest choice consists in including just an inert scalar state [5], that is, a scalar particle only interacting with the Higgs boson (and gravity) thus transforming as the singlet representation of the EW gauge group $S U(2) \times$ $U(1)$ (and similarly not charged under the color group) and which acquire no vacuum expectation value. Such a choice minimizes unwanted effects on EW radiative corrections and other physics well described by the standard model (SM).

If in addition we impose a $Z_{2}$ symmetry under which the inert scalar is odd and all the SM fields are even, the new state will couple to the SM Higgs doublet only through quartic interactions in the scalar potential. By construction, we only look for solutions with vanishing vacuum expectation value, thus $Z_{2}$ is unbroken and after EW symmetry breaking the singlet state can, as we shall discuss, potentially be a viable cold dark matter (DM) candidate.

The little hierarchy problem is often discussed in terms of the quadratic divergence arising in the mass term of the Higgs boson in a momentum dependent regularization (or, equivalently, in a pole in $d=2$ dimensions in dimensional regularization). In the past they have been cancelled either by assuming a symmetry (usually, supersymmetry) or by assuming that the Veltman condition [6] is satisfied, namely that the new sector couples to the SM Higgs boson just so as to make the one-loop quadratic divergences to the SM Higgs boson mass vanish (see [7] for various applications of this idea). These divergent terms are a different and independent problem from the one discussed here which only depends on integrating out the heavy modes in the low-energy effective theory. The terms we worry about are finite terms similar to those arising in a supersymmetric theory with soft mass terms where the quadratic divergencies are cancelled while, after integrating out the heavy states, there are finite terms 
whose contribution shifts the values of the Higgs boson mass.

2. The first SM extension we consider is a representative see-saw model [8] for the neutrino masses. Three right-handed neutrinos $N_{i}$ are added. The lagrangian of the model is given by the kinetic and Yukawa terms of the SM with the addition of the neutrino Yukawa terms:

$$
\mathcal{L}=\mathcal{L}_{Y_{S M}}+y_{i j}^{\nu} \bar{N}_{i} \tilde{H}^{\dagger} L_{j}+\frac{1}{2} M_{N_{i}} N_{i} N_{i}
$$

We work in the basis in which the right-handed neutrino mass matrix is real and diagonal.

We compute the one-loop finite contributions to the Higgs boson mass using dimensional regularization with renormalization scale $\mu$. The SM particle contributions are negligible. To compute the one-loop contribution arising from the right-handed neutrinos we rotate the Yukawa couplings $y_{i j}^{\nu}$ into the basis in which the neutrino mass matrix, defined as

$$
m_{\nu}=-y^{\nu T} \cdot \frac{1}{M_{N}} \cdot y^{\nu} v_{W}^{2},
$$

is diagonal. According to the Casas-Ibarra parametrization [9] we have that

$$
\hat{y}_{i j}^{\nu}=\left(y^{\nu} U\right)_{i j}=M_{N_{i}}^{1 / 2} R_{i j}^{\dagger} \hat{m}_{\nu_{j}}^{1 / 2},
$$

where $\hat{m}_{\nu}$ is the light neutrino diagonal mass matrix and $R$ an arbitrary orthogonal complex matrix.

In the traditional see-saw model the Yukawa couplings are of order one and the masses $M_{N_{i}}$ very large and close to the GUT scale. If the Yukawa couplings are taken to be small, the $M_{N_{i}}$ can be accordingly lighter.

Taking into account the one-loop contribution, and assuming right-handed neutrino degeneracy as well as $R$ real, the Higgs boson mass receives a shift given by

$$
\frac{1}{16 \pi^{2}} \frac{M_{N}^{3}}{v_{W}^{2}} \sum m_{\nu}\left(\frac{3}{2}-\log \frac{M_{N}^{2}}{\mu^{2}}\right),
$$

being $\mu$ the matching scale that in this case we identify with $M_{N}$. The sum of the neutrino masses, the term $\sum m_{\nu}$ in eq. (4), has a lower bound of about $0.055 \mathrm{eV}$ [10], which corresponds to a normal neutrino mass hierarchy with vanishing lightest mass. On the other hand, cosmological constraints set an upper bound on $\sum m_{\nu}$ that, even if model dependent, is always $\leq 0.44 \mathrm{eV}$ [11].

Because of the smallness of the neutrino mass term, as long as the new states have masses up to around $10^{4}$ $\mathrm{TeV}$, the shift in the Higgs boson mass is of the order of its mass and no hierarchy problem arises. Notice that the one-loop correction of right-handed neutrinos with $M_{N} \sim 10^{4} \mathrm{TeV}$ gives rise to a correction to the Higgs boson mass of the order of

$$
\left(\frac{\sqrt{M_{N} \sum m_{\nu}}}{v_{W}}\right) M_{N} \sim 2.5 \mathrm{TeV},
$$

for which also two-loop corrections are under control.

On the other hand, if the new fermion masses $M_{N_{i}} \simeq$ $M_{N}$ are larger than $10^{4} \mathrm{TeV}$, we do have a little hierarchy problem and must balance their one-loop contribution against some other contribution in order to keep the overall renormalization of the Higgs boson mass of the order of the weak scale.

To provide for such a contribution, we add the simplest state: an inert scalar particle $S$. The scalar potential is given by

$$
\begin{aligned}
V(H, S) & =\mu_{H}^{2}\left(H^{\dagger} H\right)+\mu_{S}^{2} S^{2} \\
& +\lambda_{1}\left(H^{\dagger} H\right)^{2}+\lambda_{2} S^{4}+\lambda_{3}\left(H^{\dagger} H\right) S S .
\end{aligned}
$$

Linear and trilinear terms for $S$ are absent due to the $Z_{2}$ symmetry mentioned above.

Taking into account the one loop contribution induced by the scalar state $S$ the overall shift to $\mu_{H}^{2}$, taking $\mu=M_{S}$ to minimize the logarithmic contributions to the matching, becomes

$$
\begin{aligned}
\delta \mu_{H}^{2}\left(M_{S}\right) & =\frac{1}{16 \pi^{2}}\left[-\lambda_{3} M_{S}^{2}\right. \\
& \left.-\frac{M_{N}^{3}}{v_{w}^{2}} \sum m_{\nu}\left(\log \frac{M_{N}^{2}}{M_{S}^{2}}-\frac{3}{2}\right)\right] .
\end{aligned}
$$

We want the correction in eq. (7) to be of the order of the Higgs boson mass itself. For simplicity, we can just impose that $\delta \mu_{H}^{2}=0$ and obtain

$$
\lambda_{3}=\frac{3}{2}\left(\frac{M_{N}^{3} \sum m_{\nu}}{M_{S}^{2} v_{W}^{2}}\right)\left[1-\frac{4}{3} \log \frac{M_{N}^{2}}{M_{S}^{2}}\right]
$$

In the region $M_{S} \ll M_{N}, M_{N}>10^{4} \mathrm{TeV}$ a cancellation is possible provided $\lambda_{3}$ is negative. $\lambda_{3}$ is bounded by

$$
\lambda_{3} \geq-2 \sqrt{\lambda_{1} \lambda_{2}},
$$

to ensure the stability of the scalar potential at infinity. The value of $\lambda_{1}$ is fixed by the value of the Higgs boson mass to be $\lambda_{1} \sim$.13. Eq. (8) and the above condition are satisfied for $M_{S}>5 \mathrm{TeV}$.

For $M_{S}$ around $10 \mathrm{TeV}, \lambda_{3} \simeq 0.2$. As the value of $M_{S}$ comes close to that of $M_{N}$-and the logarithmic term becomes smaller - the value of $\lambda_{3}$ becomes positive and smaller; it is of the order of $10^{-7}$ for $M_{S} \simeq M_{N}$.

The order of the the one-loop contribution to the Higgs boson mass is $\sqrt{\lambda_{3}} M_{S}$. The two-loop contributions are under control as long as this correction is $\sim 10 \mathrm{TeV}$.

3. The second SM extension we discuss is one in which we introduce the minimal set of fermion providing gauge coupling unification. The same question has been addressed in the context of split-supersymmetry models $[12$. The possible sets are given by $(Q+\bar{Q})+(D+\bar{D})$, two chiral couples of left-handed fermions with quantum number identical to the left-handed quark doublet and right-handed down quark respectively, or by 
$(L+\bar{L})+V+G$, one chiral couple of left-handed leptonlike fermion and a wino-like as well as a gluino-like fermion multiplets. We choose the first option as the minimal and representative set. They couple to the Higgs boson SM through the Yukawa lagrangian

$$
M_{Q} \bar{Q} Q+M_{D} \bar{D} D+k_{1} \bar{Q} D H+k_{2} \bar{D} Q H^{*},
$$

and they give a shift to the Higgs boson mass equal to

$$
\frac{|k|^{2}}{16 \pi^{2}}\left[\left(3 M_{Q}^{2}-M_{Q} M_{D}\right)-3 M_{Q} M_{D} \log \frac{M_{Q}^{2}}{\mu^{2}}\right],
$$

with $|k|^{2}=\left|k_{1}\right|^{2}+\left|k_{2}\right|^{2}$ and $M_{Q} \sim M_{D}$. We identify the the matching scale $\mu$ with $M_{D}$.

If the new fermions are lighter than $1 \mathrm{TeV}$ there is no little hierarchy problem. On the other hand, if they are heavier the problem exists and we introduce a inert singlet scalar $S$ to protect the Higgs boson mass. Therefore, we add the terms

$$
k_{3_{i}} \bar{Q} q_{i} S+k_{4_{i}} d_{L_{i}}^{c} D S+H . c .-V(H, S)
$$

to the lagrangian eq. (10). In eq. (12) $V(H, S)$ coincides with eq. (6) with $S$ odd under an additional $Z_{2}$ symmetry. We have also imposed for extra fermions to be odd under $Z_{2}$. The total one-loop contribution to $\mu_{H}^{2}$ at the scale $\mu=M_{S}$ is given by

$$
\begin{aligned}
\delta \mu_{H}^{2}\left(M_{S}\right) & =\frac{1}{16 \pi^{2}}\left[-\lambda_{3} M_{S}^{2}+|k|^{2}\left(3 M_{Q}^{2}-M_{Q} M_{D}\right)\right. \\
& \left.-3|k|^{2} M_{Q} M_{D} \log \frac{M_{Q}^{2}}{M_{S}^{2}}\right]
\end{aligned}
$$

Let us consider the case in which all couplings are of order one. As before, for simplicity, we just impose that $\delta \mu_{H}^{2}=0$. Taking $M_{Q} \sim M_{S}$ and with the singlet $S$ lightest $Z_{2}$-odd particle, this condition is satisfied by writing $\lambda_{3}$ as a function of $|k|^{2}$ :

$$
\lambda_{3}=|k|^{2}\left(\frac{M_{Q}}{M_{S}}\right)^{2}\left(2-3 \log \frac{M_{Q}^{2}}{M_{S}^{2}}\right) \sim 2|k|^{2} .
$$

Contrary to the previous example of the see-saw model, in this case it is always possible to find an appropriate value of $\lambda_{3}$ so as to control the renormalization of the Higgs boson mass.

4. We may ask whether in the two models considered the inert scalar $S$ is a viable DM candidate. It is a gauge singlet and therefore only interacts with the SM particles through the Higgs boson $h$. The point-like interaction $\lambda_{3} / 2 S S h h$ and the scattering mediated by $h$-both in the $s$ and $t$ channels - contribute to the cross section $S S \rightarrow h h$. The Higgs boson $h$ also mediates the scattering processes $S S \rightarrow f \bar{f}, S S \rightarrow W^{+} W^{-}, S S \rightarrow Z Z$.

It has been shown [13] that a single inert singlet that couples with the Higgs boson with a small coupling is a realistic cold $\mathrm{DM}$ candidate with a mass $\lesssim v_{W}$. In our case, the singlet may account for the correct relic density in the opposite regime where its mass is $\gg v_{W}$ and its coupling with the Higgs boson relatively large. In this case, the scattering amplitude is dominated by the pointlike $S S \rightarrow h h$ vertex which gives a contribution to the total cross section equal to

$$
\langle\sigma v\rangle \simeq \frac{1}{16 \pi} \frac{\lambda_{3}^{2}}{M_{S}^{2}}
$$

To estimate the viability of $S$ as DM candidate, we make use of the approximated analytical solution [14]. The relic abundance $n_{\mathrm{DM}}$ is written as

$$
\frac{n_{\mathrm{DM}}}{s}=\sqrt{\frac{180}{\pi g_{*}}} \frac{1}{M_{p l} T_{f}\langle\sigma v\rangle},
$$

where $M_{p l}$ is the Planck mass, $T_{f}$ is the freeze-out temperature, which for our and similar candidates is given by $m_{S} / T_{f} \sim 26$. The constant $g_{*}=106.75+1$ counts the number of SM degrees of freedom in thermal equilibrium plus the additional degrees of freedom related to the singlets, $s$ is their total entropy density. Current data fit within the standard cosmological model give a relic abundance with $\Omega_{\mathrm{DM}} h^{2}=0.112 \pm 0.006$ [15] which corresponds to

$$
\frac{n_{\mathrm{DM}}}{s}=\frac{(0.40 \pm 0.02)}{10^{9} M_{S} / \mathrm{GeV}} .
$$

By combining eq. (17) with eq. (15) we may write $\lambda_{3}$ as function of $M_{S}$ obtaining

$$
\left|\lambda_{3}\right| \simeq 0.44 \frac{M_{S}}{\mathrm{TeV}}
$$

In the first model we considered, the condition eq. 18 can only be satisfied in the case in which $M_{S} \ll M_{N}$, as it is shown in Fig. 1. For $M_{S} \simeq M_{N}$, the smallness of the neutrino Yukawa couplings forces $\lambda_{3}$ to be very small thus destroying its potential role as DM candidate. More dangerously, in the latter case, it could give rise to the overclosure of the universe. Since its production mechanism could be non-thermal any conclusion should be drawn only after a detailed analysis that goes beyond the purposes of this work. In any case, we could let $S$ acquire a small vacuum expectation value $\sim v_{W}^{2} / M_{S}$ and not impose the $Z_{2}$ symmetry so that the scalar state would rapidly decay into SM particles through its mixing with the SM Higgs boson.

In the second model we discussed, $\lambda_{3}$ depends only on the ratio $M_{Q} / M_{S}$ and it is scale independent, thus the correct relic density may be accommodated for any value of $M_{S}$, in particular for $M_{S} \geq 10 \mathrm{TeV}$.

5. Let us briefly comment on the possibility of detecting the inert scalar $S$ in nuclear scattering experiments. 


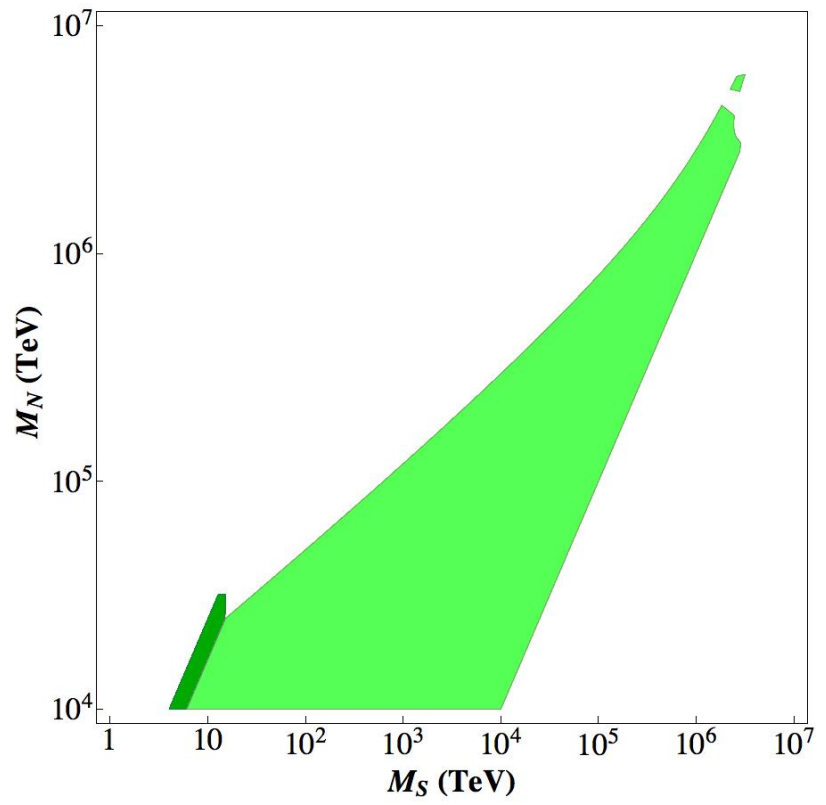

FIG. 1: See-saw model: The two regions for which the oneloop contribution vanishes and $\lambda_{3}$ satisfy eq. 18) (narrow dark green region) and $\left|\lambda_{3}\right|<0.44 M_{S} / \mathrm{TeV}$ (light green region) respectively. The points have been selected by requiring that: $\lambda_{3}>-1.6$ to avoid too a large a value for $\lambda_{2}$, according to eq. (9), the order of $\sqrt{\lambda_{3}} M_{S} \lesssim 10 \mathrm{TeV}$ to control the twoloop corrections, $M_{N} \geq M_{S}$ according to our assumption and $\sum m_{n} u$ in the range $0.055-0.44 \mathrm{eV}$ (see the text). For the points in the narrow dark-green region, the model provide a viable DM candidate, whereas for those in the light-green region a detailed analysis of the singlet production mechanism should be done before ruling out the model, as commented in the text.

The $\lambda_{3}$ quartic term in eq. (6) gives rise also to the three fields interaction $S S h$ which yields the effective singlet-nucleon vertex

$$
f_{N} \frac{\lambda_{3} m_{N}}{m_{h}^{2}} S S \bar{\psi}_{N} \psi_{N} .
$$

The (non-relativistic) cross section for the process is given by [17]

$$
\sigma_{N}=f_{N}^{2} m_{N}^{2} \frac{\lambda_{3}^{2}}{4 \pi}\left(\frac{m_{r}}{m_{S} m_{h}^{2}}\right)^{2}
$$

where $m_{r}$ is the reduced mass for the system which is, to a vary good approximation in our case, equal to the nucleon mass $m_{N}$; the factor $f_{N}$ contains many uncertainties due to the computation of the nuclear matrix elements and it can vary from 0.3 to 0.6 [18. Substituting the values we have found for our model, we obtain, depending on the choice of parameters within the given uncertainties, a cross section $\sigma_{N}$ between $10^{-45}$ and $10^{-44} \mathrm{~cm}^{2}$, a value within reach of the next generation of experiments (see Fig. 2).

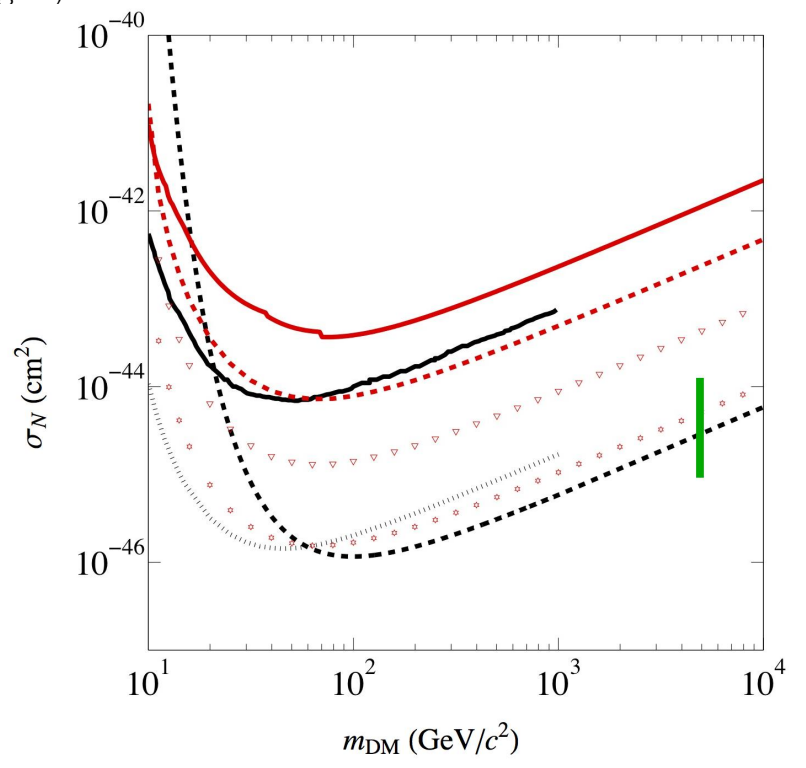

FIG. 2: Spin independent cross section per nucleon versus DM candidate masses 19. The black (red) solid line corresponds to the XENON100 (CDMSII) data. Black points and the black dashed line are the projections for upgraded XENON100 and XENON1T, respectively. The red dashed line, down triangles and stars correspond to different projections for SCDMS. The green vertical line is the prediction of the inert model discussed in this work.

6. As the scale of new physics is pushed to around the $10 \mathrm{TeV}$ scale or higher, the stability of the Higgs boson mass against finite one-loop corrections induced by the new states give rise to a little hierarchy problem. Since these new states are beyond the current experimental reach, we can use this problem in an heuristic manner to determine masses and couplings of the new particles. We have shown that for two representative new physics scenarios - namely, see-saw neutrino mass generation and gauge couplings unification - the addition of an inert scalar state suffices in solving the little hierarchy problem and provides in addition a viable candidate for DM. Such a candidate may well be the only experimentally testable signature of the new physics.

\section{Acknowledgments}

We thank P. Ullio for explaining to us some aspects of DM physics. MF thanks SISSA for the hospitality. 
[1] G. Aad et al. [ATLAS Collaboration], ATLAS-CONF2012-109 and ATLAS-CONF-2011-132 arXiv:1109.6572 [hep-ex]]; S. Chatrchyan et al. [CMS Collaboration], arXiv:1109.2352, [hep-ex] 1107.5834 [hep-ex] and CMSPAS-HIG-11-020 and CMS-PAS-SUS-11-022.

[2] R. Barbieri and A. Strumia, hep-ph/0007265 and Phys. Lett. B 462, 144 (1999) hep-ph/9905281.

[3] C. Balazs, A. Buckley, D. Carter, B. Farmer and M. White, arXiv:1205.1568 [hep-ph]; O. Buchmueller, R. Cavanaugh, M. Citron, A. De Roeck, M. J. Dolan, J. R. Ellis, H. Flacher and S. Heinemeyer et al., Eur. Phys. J. C 72, 2243 (2012) arXiv:1207.7315 [hep-ph]]; S. Akula, P. Nath and G. Peim, Phys. Lett. B 717, 188 (2012) arXiv:1207.1839 [hep-ph]]; A. Fowlie, M. Kazana, K. Kowalska, S. Munir, L. Roszkowski, E. M. Sessolo, S. Trojanowski and Y. -L. S. Tsai, Phys. Rev. D 86, 075010 (2012) arXiv:1206.0264 [hep-ph]]; C. Strege, G. Bertone, F. Feroz, M. Fornasa, R. R. de Austri and R. Trotta, arXiv:1212.2636 [hep-ph]; M. E. Cabrera, J. A. Casas and R. R. de Austri, arXiv:1212.4821 [hep$\mathrm{ph}$.

[4] G. Aad et al. [ATLAS Collaboration], Phys. Lett. B710, 49 (2012) arXiv:1202.1408 [hep-ex]] and ATLAS-CONF2012-093; S. Chatrchyan et al. [CMS Collaboration], Phys. Lett. B 710, 26 (2012) arXiv:1202.1488[hep-ex]]S. Chatrchyan et al. [CMS Collaboration], arXiv:1202.1488 [hep-ex] and CMS-PAS-HIG-12-020.

[5] N. G. Deshpande and E. Ma, Phys. Rev. D 18, 2574 (1978); R. Barbieri, L. J. Hall and V. S. Rychkov, Phys. Rev. D 74, 015007 (2006) hep-ph/0603188.

[6] M. J. G. Veltman, Acta Phys. Polon. B 12, 437 (1981); see, also, P. Osland and T. T. Wu, Phys. Lett. B 291, 315 (1992); E. Ma, Phys. Rev. D 47, 2143 (1993) hepph/9209221; G. Ossola and A. Sirlin, Eur. Phys. J. C 31, 165 (2003) hep-ph/0305050.

[7] A. Kundu and S. Raychaudhuri, Phys. Rev. D 53, 4042 (1996) hep-ph/9410291; N. G. Deshpande, R. J. Johnson and E. Ma, Phys. Lett. B 130, 61 (1983); F. Bazzocchi, M. Fabbrichesi and P. Ullio, Phys. Rev. D 75, 056004 (2007) hep-ph/0612280; B. Grzadkowski and J. Wudka, Phys. Rev. Lett. 103, 091802 (2009) arXiv:0902.0628 [hep-ph]]; A. Drozd, B. Grzadkowski and J. Wudka, JHEP 1204, 006 (2012) arXiv:1112.2582 [hep-ph]]; F. Bazzocchi and M. Fabbrichesi, arXiv:1207.0951] [hep$\mathrm{ph}$.

[8] P. Minkowski, Phys. Lett. B67, 421 (1977); M. Gell-
Mann, P. Ramond and R. Slansky, (1979), Print-800576 (CERN); T. Yanagida, (KEK lectures, 1979), ed. Sawada and Sugamoto (KEK, 1979); R. N. Mohapatra and G. Senjanovic, Phys. Rev. Lett. 44, 91 (1980). J. Schechter and J. W. F. Valle, Phys. Rev. D22, 2227 (1980); Phys. Rev. D25, 774 (1982); G. Lazarides, Q. Shafi and C. Wetterich, Nucl. Phys. B181, 287 (1981).

[9] J. A. Casas and A. Ibarra, Nucl. Phys. B 618, 171 (2001) arXiv:hep-ph/0103065.

[10] D. V. Forero, M. Tortola and J. W. F. Valle, Phys. Rev. D 86 (2012) 073012 arXiv:1205.4018 [hep-ph]]; G. L. Fogli, E. Lisi, A. Marrone, D. Montanino, A. Palazzo and A. M. Rotunno, Phys. Rev. D 86, 013012 (2012) arXiv:1205.5254 [hep-ph]]; M. C. Gonzalez-Garcia, M. Maltoni, J. Salvado and T. Schwetz, arXiv:1209.3023 [hep-ph].

[11] M. C. Gonzalez-Garcia, M. Maltoni and J. Salvado, JHEP 1008, 117 (2010) arXiv:1006.3795 [hep-ph]].

[12] G. F. Giudice and A. Romanino, Nucl. Phys. B 699, 65 (2004) [Erratum-ibid. B 706, 65 (2005)] hep$\mathrm{ph} / 0406088$.

[13] J. McDonald, Phys. Rev. D 50, 3637 (1994) hepph/0702143 ; C. P. Burgess, M. Pospelov and T. ter Veldhuis, Nucl. Phys. B 619, 709 (2001) hep-ph/0011335; R. Dick, R. B. Mann and K. E. Wunderle, Nucl. Phys. B 805, 207 (2008) arXiv:0803.1444 [astro-ph]]; C. E. Yaguna, JCAP 0903, 003 (2009) arXiv:0810.4267 [hep$\mathrm{ph}]$.

[14] M. Srednicki, R. Watkins and K. A. Olive, Nucl. Phys. B 310, 693 (1988); P. Gondolo and G. Gelmini, Nucl. Phys. B 360, 145 (1991); E.W. Kolb and M.S. Turner, The early universe, Addison-Wesley, Reading MA, 1993; M. Cirelli, N. Fornengo and A. Strumia, Nucl. Phys. B 753, 178 (2006). hep-ph/0512090.

[15] J. Beringer et al. (Particle Data Group), Phys. Rev. D86, 010001 (2012).

[16] M. Cirelli et al., JCAP 1103 (2011) 051, [arXiv 1012.4515]; P. Ciafaloni et al., JCAP 1103 (2011) 019, [arXiv 1009.0224].

[17] R. Barbieri, M. Frigeni and G. F. Giudice, Nucl. Phys. B 313, 725 (1989); J. R. Ellis et al., Phys. Rev. D 71, 095007 (2005) hep-ph/0502001.

[18] A. Bottino, et al. Astropart. Phys. 13, 215 (2000) hepph/9909228 and 18, 205 (2002) hep-ph/0111229.

[19] http://dmtools.brown.edu/ 